\title{
HPV testing as a triage for borderline or mild dyskaryosis on cervical cytology: results from the Sentinel Sites study
}

\author{
RS Kelly', J Patnick ${ }^{2}$, HC Kitchener ${ }^{3}$ and SM Moss*,I on behalf of the NHSCSP HPV Special Interest Group ${ }^{4}$ \\ 'Cancer Screening Evaluation Unit, Institute of Cancer Research, Richard Doll Building, I 5 Cotswold Road, Sutton, Surrey SM2 5NG, UK; ${ }^{2}$ NHS Cancer \\ Screening Programmes, Sheffield, UK; ${ }^{3}$ School of Cancer Studies and Enabling Sciences, University of Manchester, Manchester Academic Health \\ Science Centre, Manchester, UK
}

BACKGROUND: Earlier pilot studies of human papillomavirus (HPV) triage concluded that HPV triage was feasible and cost-effective. The aim of the present study was to study the impact of wider rollout of HPV triage for women with low-grade cytology on colposcopy referral and outcomes.

METHODS: Human papillomavirus testing of liquid-based cytology (LBC) samples showing low-grade abnormalities was used to select women for colposcopy referral at six sites in England. Samples from 1005 I women aged 25-64 years with routine call or recall cytology reported as borderline or mild dyskaryosis were included.

RESULTS: Human papillomavirus-positive rates were $53.7 \%$ in women with borderline cytology and $83.9 \%$ in those with mild dyskaryosis. The range between sites was 34.8-73.3\% for borderline cytology, and 73.4-91.6\% for mild dyskaryosis. In the single site using both LBC technologies there was no difference in rates between the two technologies. The positive predictive value of an HPV test was $16.3 \%$ for CIN2 or worse and $6.1 \%$ for CIN3 or worse, although there was considerable variation between sites. CONCLUSION: Triaging women with borderline cytological abnormalities and mild dyskaryosis with HPV testing would allow approximately a third of these women to be returned immediately to routine recall, and for a substantial proportion to be referred for colposcopy without repeat cytology. Variation in HPV-positive rates results in differing colposcopy workload.

British Journal of Cancer (20II) 1 05, 983-988. doi:I0.1038/bjc.20 I I.326 www.bjcancer.com

Published online 6 September 2011

(c) 201 I Cancer Research UK

Keywords: cervical cytology; screening; human papillomavirus; triage

The introduction of an organised cervical screening programme in the United Kingdom in 1988 has led directly to a fall in the annual number of new cases of invasive cervical cancer. The NHS cervical screening programme has been estimated to prevent up to 3900 cases of cervical cancer (Sasieni et al, 1996; NHS, 2009) and save approximately 4500 lives per year by 2030 (Peto et al, 2004).

Infection with high-risk human papillomavirus (HPV) is now known to be a necessary aetiological factor in the development of cervical cancer (Munoz et al, 2006). Those strains of HPV associated with genital tract infection are subdivided into high- and low-risk types, and of the former HPV 16 and 18 are estimated to be responsible for over $70 \%$ of all cases of cervical cancer (Howell-Jones et al, 2010). Testing liquid residue from samples with borderline cytological abnormalities or mild dyskaryosis for high-risk HPV DNA can identify those women who are at risk of disease from those who have only a negligible risk of high-grade CIN. A meta-analysis has found that the use of HPV testing for triage in this way improved the accuracy for ASCUS samples for an outcome of CIN2 or worse compared with repeat cytology (Arbyn et al, 2004).

In 2001, the HPV/liquid-based cytology (LBC) pilot studies reported on the feasibility of introducing HPV triage in the English

\footnotetext{
*Correspondence: Dr SM Moss; E-mail: sue.moss@icr.ac.uk

${ }^{4}$ See Appendix.

Received 4 May 2011; revised 20 July 2011; accepted 26 July 201।; published online 6 September 2011
}

screening programme. Three sites converted to using LBC and HPV triage with the Hybrid Capture 2 (HC2) assay for women with borderline cytology or mild dyskaryosis. Initially all HPV-positive women were referred to colposcopy, whereas HPVnegative women were re-tested at 6 months and referred to colposcopy if found to be HPV-positive or cytology of mild dyskaryosis or worse. Readings greater than three times the kit derived cutoff (Co) value in relative light units (RLUs) were considered positive ( $>3 \mathrm{RLU} / \mathrm{Co}$ ). The protocol was amended in two sites, where HPV-positive women aged under 35 years were re-tested at 6 months and only referred to colposcopy if HPV infection and/or cytological abnormality persisted. The results of the pilot study suggested that although HPV triage decreased the number of repeat cytology tests and reduced the time taken to return women to routine recall, it resulted in a large increase in referrals to colposcopy. Nevertheless, not only was HPV triage feasible and acceptable to women but also the results of an economic analysis concluded that it was cost-effective, both in terms of quality and of life years saved (Legood et al, 2006; Moss et al, 2006).

In 2007, the 'Sentinel sites' protocol was implemented, representing $\sim 10 \%$ of the English cervical screening programme. The sites included two from the original pilots (Bristol and Norfolk and Norwich), and four additional ones (Liverpool, Manchester, Sheffield and Northwick Park). An agreed protocol for the use of HPV triage for women with borderline or mild dyskaryosis was followed. 
The evaluation of this project aimed to provide information on the likely effect of national rollout, including rates of referral to colposcopy, and the positive predictive value (PPV) of this approach.

\section{MATERIALS AND METHODS}

Samples from women, aged 25-64 years, undergoing routine call or recall cytology at the six sites reported as borderline or mild dyskaryosis, were included in the protocol.

Cytology was liquid based; three sites used ThinPrep LBC (Hologic, Bedford, MA, USA), two sites used BD SurePath LBC (Beckton Dickinson, Franklin Lakes, NJ, USA) and one site used both the technologies. Samples reported as borderline or mild dyskaryosis were sent to one of two HPV testing laboratories serving all six of the sentinel sites. The Qiagen HC2 (Crawley, England) assay was used, with a cut-off of 2 RLU/Co to determine positivity (Sargent et al, 2010).

The protocol is shown in Figure 1. Women who tested negative for HPV were returned to routine recall at 3 or 5 years, depending on age; those who were HPV-positive were referred to colposcopy.
Women who appeared normal at colposcopic inspection and those found to have no CIN on biopsy were returned to routine recall. Women diagnosed with CIN1 on biopsy and who were not treated underwent a 12-month surveillance cytology. Women with CIN2 or worse were treated and followed up with a test of cure protocol. This comprised of repeat cytology at 6 months and a reflex HPV test in those with negative cytology, with referral to colposcopy if either was positive; all other women were returned to routine recall at 3 years (regardless of age). The results of the analysis of the test of cure protocol will be presented elsewhere, as will be data on costeffectiveness. Anonymous data on relevant cytology HPV tests, RLU values, biopsy, treatment and histology were collected, together with information on management and reasons for non-attendance.

The study took place between 1 January 2008 and 1 April 2009. Follow-up data were collected until September 2009, with additional data requested for those individual women with outstanding cytology and/or colposcopy outcomes. Statistical analysis was conducted using STATA version 10 (Stata Corporation, College Station, TX, USA). Age standardisation used 5-year age groups, standardised to the European standard population (Waterhouse et al, 1976).

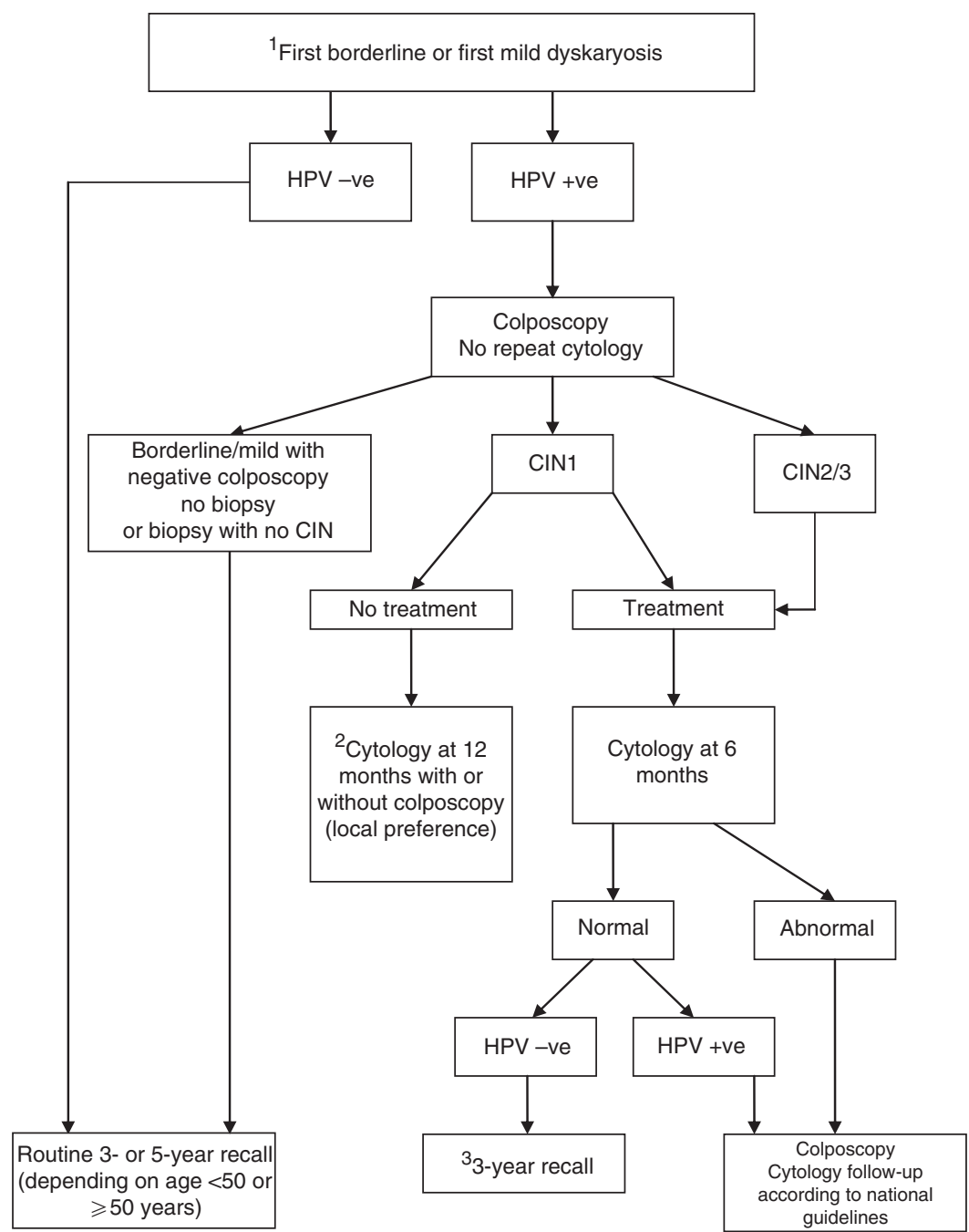

Figure I Study protocol. 'If sample is inadequate for the HPV test, recall for 6-month repeat cytology/HPV test or refer to colposcopy, depending on local referral practice. ${ }^{2}$ Follow-up after I2-month cytology only, should follow normal NHSCSP protocols. ${ }^{3}$ Women $\geqslant 50$ years who have normal cytology at 3 years will then return to 5 -yearly routine recall. NB Women who reach 65 years still require to complete the protocol, otherwise need to comply with the national guidelines. 
The epidemiological evaluation was carried out by the Cancer Screening Evaluation Unit.

\section{RESULTS}

A total of 10051 women entered the protocol; 6507 (64.7\%) had an initial borderline test and $3544(35.3 \%)$ had initial mild dyskaryosis.

\section{HPV-positive rates}

Of these 10051 women, 6470 (64.4\%) tested positive for high-risk HPV, $53.7 \%$ of those with a borderline test and $83.9 \%$ of those with mild dyskaryosis. There was a highly significant decreasing trend in HPV-positive rate with increasing age $(P<0.0001)$ in both categories. Less than $2 \%$ of HPV-positive women had an RLU value of between 2 and 3 . The profiles of the study participants are shown in Table 1.

The HPV-positive rates at the six sites ranged from $34.8 \%$ to $73.3 \%$ for women with borderline cytology, and from $73.4 \%$ to $91.6 \%$ for women with mild dyskaryosis. (Table 2) These differences remained after the rates were standardised for age. Overall the HPV-positive rate was higher in sites using ThinPrep LBC than in those using BD SurePath LBC; $68.7 \%$ and $61.7 \%$, respectively, $(P<0.0001)$. The difference remained after adjustment for age group and initial cytology result. LBC technology was, however, confounded by site, and it was therefore not possible to determine whether this difference was due to variation in the reporting of cytology between sites. In the only site which used both technologies there was no significant difference in positive rates between the two.

\section{Colposcopy following a positive HPV test}

At least 6 months follow-up was available for all 6470 HPVpositive women, of which 5838 (90.2\%) attended colposcopy.
Attendance varied from $96.2 \%$ to $81.4 \%$ between sites. No information was available on the remaining 632 who failed to attend.

Table 3 shows the outcome at colposcopy in the 5838 women who attended. The proportion of women who were negative at colposcopy, due either to a negative biopsy or to a negative colposcopic assessment, resulting in no biopsy being performed, was significantly higher in women with initial borderline cytology than in women with mild dyskaryosis, $59.9 \%$ and $48.3 \%$, respectively, $(P<0.0001)$. Overall, $29 \%$ of these had no biopsy.

There were 298 colposcopies for which information on biopsy type was not available or was coded as 'other'. Nearly 19\% (1093 out of 5838) of women who attended colposcopy were reported as not undergoing biopsy, although $16.0 \%$ (175 out of 1093) of these women had a recorded diagnosis of CIN1. Almost 63\% (3631 out of 5838) of all women who attended colposcopy underwent a diagnostic punch biopsy, of which $49.0 \%$ (1778 out of 5838) were negative for CIN. Excision biopsy by LLETZ was reported in 196 women, of whom only $114(58.2 \%)$ were found to have CIN2 or worse, and almost 30\% (56) were negative for CIN. A further 442 women were known to have had a biopsy, but the type was not specified.

The type of procedure performed at colposcopy varied greatly by centre, with the percentage of women not undergoing biopsy varying from $65.9 \%$ to $2.7 \%$, and the proportion undergoing punch biopsy varying from $26.5 \%$ to $95 \%$.

\section{Positive predictive value}

The PPV of a positive HPV test for detecting high-grade CIN in women who attended colposcopy was $6.1 \%$ for CIN3 or worse and $16.3 \%$ for CIN2 or worse (Table 3 ). The PPV for CIN3 or worse was slightly, but significantly, higher in women with initial borderline cytology than in women with initial mild dyskaryosis $(6.7 \% v s$ $5.4 \% ; P=0.03$ ); but this was not observed for CIN2 or worse. There was a highly significant decreasing trend in the PPV for CIN2 or

Table I HPV-positive rates by age group and initial cytology result

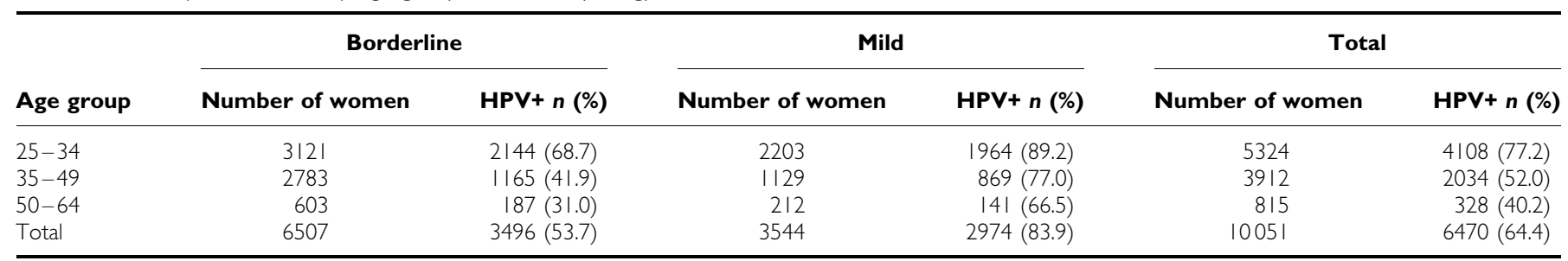

Abbreviation: HPV = human papillomavirus.

Table 2 HPV-positive rates by site and initial cytology

\begin{tabular}{|c|c|c|c|c|c|c|}
\hline \multirow[b]{2}{*}{ Site } & \multicolumn{2}{|c|}{ Borderline } & \multicolumn{2}{|c|}{ Mild } & \multicolumn{2}{|c|}{ Total } \\
\hline & $n$ & HPV +ve (\%) & $n$ & HPV +ve (\%) & $n$ & HPV +ve (\%) \\
\hline$A$ & 789 & $455(57.7)$ & 420 & $372(88.6)$ & 1209 & $827(68.4)$ \\
\hline$B$ & 643 & $224(34.8)$ & 523 & $384(73.4)$ & 1166 & $608(52.1)$ \\
\hline C & 2557 & |||| (43.4) & 1507 & $1232(81.8)$ & 4064 & $2343(57.7)$ \\
\hline $\mathrm{D}$ & 663 & $406(61.2)$ & 557 & $500(89.8)$ & 1220 & $906(74.3)$ \\
\hline E & 1263 & $866(68.6)$ & 404 & $370(91.6)$ & 1667 & $1236(74.1)$ \\
\hline $\mathrm{F}$ & 592 & $434(73.3)$ & 133 & $116(87.2)$ & 725 & $550(75.9)$ \\
\hline C (Thinprep LBC) & 1188 & $543(47.7)$ & 798 & $669(83.8)$ & 1986 & $1212(61.0)$ \\
\hline C (BD Surepath LBC) & 426 & $216(50.7)$ & 241 & $204(84.6)$ & 667 & $420(63.0)$ \\
\hline Total (Thinprep LBC) & 3903 & $2270(58.2)$ & 2179 & 1911 (87.7) & 6082 & $4181(68.7)$ \\
\hline Total (BD Surepath LBC) & $|66|$ & $874(52.6)$ & 897 & 704 (78.5) & 2558 & $1578(61.7)$ \\
\hline
\end{tabular}

Abbreviations: HPV = human papillomavirus; LBC = liquid-based technology. Technology not known for 943 borderline and 468 mild samples. 
Table 3 Results of follow-up of HPV-positive women

\begin{tabular}{|c|c|c|c|c|c|c|c|}
\hline & $\begin{array}{c}\text { Inadequate/unknown/ } \\
\text { other, } n(\%)\end{array}$ & Negative, $n$ (\%) & $\begin{array}{c}\text { Positive } \\
\text { cytology, } n \text { (\%) }\end{array}$ & CINI, n (\%) & CIN2, n (\%) & CIN3+, n (\%) & Total, $n$ (\%) \\
\hline \multicolumn{8}{|l|}{$B L$} \\
\hline $25-34$ & $64(3.3)$ & $109 \mid(56.6)$ & $45(2.3)$ & $394(20.4)$ & $193(10.0)$ & $142^{\mathrm{a}}(7.4)$ & $1929(100.0)$ \\
\hline $50-64$ & $8(4.7)$ & $124(72.5)$ & $3(1.8)$ & $27(15.8)$ & $4(2.3)$ & $5(2.9)$ & $171(100.0)$ \\
\hline Total & $102(3.2)$ & $1894(59.9)$ & $74(2.3)$ & $594(18.8)$ & $285(9.0)$ & $212(6.7)$ & $3161(100.0)$ \\
\hline \multicolumn{8}{|l|}{ Mild } \\
\hline $35-49$ & $27(3.4)$ & $392(49.2)$ & $25(3.1)$ & $236(29.6)$ & $87(10.9)$ & $30(3.8)$ & $797(100.0)$ \\
\hline $50-64$ & $3(2.3)$ & $80(61.5)$ & $5(3.8)$ & $31(23.8)$ & $8(6.2)$ & $3(2.3)$ & $130(100.0)$ \\
\hline Total & $101(3.8)$ & $1293(48.3)$ & $92(3.4)$ & $735(27.5)$ & $312(11.7)$ & $144(5.4)$ & $2677(100.0)$ \\
\hline Total & $203(3.5)$ & $3187(54.6)$ & $166(2.8)$ & 1329 (22.8) & $597(10.2)$ & $356(6.1)$ & $5838(100.0)$ \\
\hline
\end{tabular}

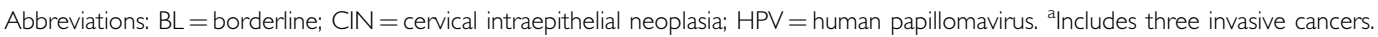

Table 4 PPV of colposcopy by site

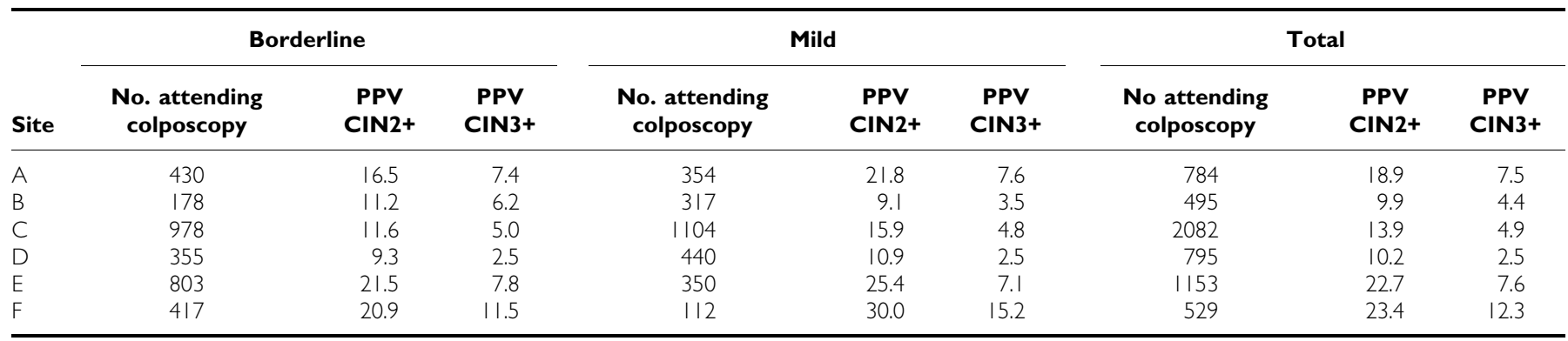

Abbreviations: $\mathrm{CIN}=$ cervical intraepithelial neoplasia; $\mathrm{PPV}=$ positive predictive value.

worse with increasing age group $(P<0.0001)$, which was observed both in women with initial borderline and initial mild dyskaryosis.

The PPV of HPV for detecting high-grade disease varied by centre (Table 4); the PPV for CIN2 or worse ranged from $9.3 \%$ to $21.5 \%$ and for CIN3 or worse from $2.5 \%$ to $11.5 \%$ for women with borderline changes at cytology. For women with mild dyskaryosis, the PPV for CIN2 or worse ranged from $9.1 \%$ to $33.0 \%$ and for CIN3 or worse from $2.5 \%$ to $15.2 \%$. Standardising for age and for the ratio of borderline to mild tests did not reduce this variation.

\section{DISCUSSION}

This study is indicative of the impact of rolling out of HPV triage, in terms of colposcopy referral and detection of CIN $2+$. The principal findings are that referral rates following triage varied across clinical sites, and the proportion of referred women with CIN2 + (PPV) varied. The strength of this study was its real-life response to HPV triage as routine NHS practice across six sites in England. The main limitation of this study was that its observational nature resulted in a number of women not being managed according to protocol, and follow-up was, therefore, incomplete at the time of end of data collection. We believe, however, that the results for the large majority of women who did attend following referral are representative of the cohort as a whole.

The HPV-positive proportion in the current study was higher than that observed in the pilot studies of 2003-2004. As a result, referral to colposcopy of $64 \%$ was significantly higher than that observed with both the initial (48\%) and the revised (38\%) protocol of the pilot studies, despite women who were HPVnegative being returned immediately to routine recall. The PPVs of HPV for CIN2 or worse and CIN3 or worse were lower in the present study than those observed in the earlier pilot studies; $6.1 \%$ vs $8.3 \%$ for $\mathrm{CIN} 3+(P=0.004)$ and $16.3 \%$ vs $20.0 \%$ for CIN2 + $(P=0.0014)$. The attendance rate for colposcopy was high $(90.2 \%)$, and greater than that of $72 \%$ seen in a similar UK-based study for women with a borderline cytology result, a positive HPV test or both (Cuzick et al, 2003). The higher HPV-positive rates observed in this study are not readily explained by the lower cut-off for a positive test of $2 \mathrm{RLU} / \mathrm{Co}$ compared with $3 \mathrm{RLU} / \mathrm{Co}$ in the earlier studies, as the proportion of women with RLU/Co between 2.0 and 3.0 was small. They may be due, in part, to changes in the demographics of the populations, and changes in sampling technique or perhaps more likely, due to cytological classification. The HPV-positive rates observed in this study, overall, are also higher than those observed in other studies. In the UK-based ARTISTIC trial, which had a high rate of borderline cytology, the HPV-positive rate was $31.1 \%$ for borderline cytology and $69.9 \%$ for mild dyskaryosis (Kitchener et al, 2006). A meta-analysis (Arbyn et al, 2009) found an average HPV-positive rate of $43 \%$ (95\% CI: $40-46 \%$ ) for women with ASCUS/ASC-US (broadly comparable to borderline cytology) and 76\% (95\% CI: 71-81\%) for women with LSIL (broadly comparable to mild dyskaryosis).

Of importance was the observed difference between sites in HPV-positive rates. The threshold used to determine borderline and mild dyskaryosis test results will vary between laboratories. According to a 3-year average from the NHSCSP screening returns (KC61 part B: 2006-07, 2007-08 and 2008-09), the two sites with the lowest rate of HPV-positive tests classified a higher percentage of all samples taken as borderline or worse than do the other sites, suggesting that they may be including samples in their borderline changes category that other laboratories would class as negative.

Heterogeneity in HPV-positive rates between studies has also been observed in a meta-analysis of studies of triage in women with ASCUS/LSIL cytology (Arbyn et al, 2009). The rates in the current study are within the observed range of this meta-analysis. 
Both HPV testing laboratories participated in external quality control provided by the National External Quality Assessment Service and by the Scottish HPV reference laboratory, in addition to internal quality assurance (QA) procedures with retesting of $2 \%$ of all samples. There was variation in the PPV between the sites tested by each HPV testing laboratory.

The lower PPV values observed in this study may be a consequence of higher HPV-positive rates, if these are not related to a higher prevalence of CIN. The differences between sites may reflect the different rates of cytological abnormality and HPV positivity in the sites participating. Because this was a pragmatic study, there was no additional QA of the colposcopy, but all colposcopists taking part are BSCCP certified and take part in the NHSCSP QA programme. There was considerable variation in rates of punch biopsy, which implies variation in local practice and in part explains the variation in rates of high-grade CIN. In $29 \%$ (997 out of 3187) of the colposcopy results reported as negative, no biopsy was taken, but there is no evidence that the variation in PPV between sites is related to the variation in biopsy rate.

The variation in the PPV of a positive HPV test is also reflected in the literature; estimates range from a PPV of $8.3 \%$ (Guyot et al, 2003) to 58.1\% (Rebello et al, 2001) for ASCUS and CIN2 + and $8.4 \%$ (Bergeron et al, 2000) to $54.5 \%$ (Rebello et al, 2001) for LSIL and CIN2 + . A meta-analysis has found a pooled PPV of $22.3 \%$ for ASCUS and 27.3\% for LSIL (Arbyn et al, 2005). The PPV of an HPV test for high-grade disease appears more dependent on age than on the grade of cytology, which may be indicative that highrisk HPV is more important than whether a sample is classified as borderline or mild.

The results of the original pilot studies suggested that referring all HPV-positive women to colposcopy led to an earlier detection of CIN2 + compared with standard practice, however this effect was not observed for CIN3 + . The revised protocol for women aged 25-34 years adopted for part of these studies reduced the colposcopy workload somewhat, but $75 \%$ of women remained HPV-positive at 6 months, and such a policy increased the number of repeat smears and the risk of loss to follow-up. The UK-based TOMBOLA trial (TOMBOLA Group, 2009a, b, c) recommended a policy of surveillance rather than triage for two reasons; the first was that some CIN2 + will regress with time and the second was that they identified a high proportion of HPV-negative CIN 2. HPV-negative CIN2 is, however, probably of little clinical significance. The high rate of compliance with colposcopy

\section{REFERENCES}

Arbyn M, Buntinx F, Van Ranst M, Paraskevaidis E, Martin-Hirsch P, Dillner J (2004) Virologic versus cytologic triage of women with equivocal Pap smears: a meta-analysis of the accuracy to detect highgrade intraepithelial neoplasia. J Natl Cancer Inst 96(4): 280-293

Arbyn M, Martin-Hirsch P, Buntinx F, Van RM, Paraskevaidis E, Dillner J (2009) Triage of women with equivocal or low-grade cervical cytology results: a meta-analysis of the HPV test positivity rate. J Cell Mol Med 13(4): $648-659$

Arbyn M, Paraskevaidis E, Martin-Hirsch P, Prendiville W, Dillner J (2005) Clinical utility of HPV-DNA detection: triage of minor cervical lesions, follow-up of women treated for high-grade CIN: an update of pooled evidence. Gynecol Oncol 99(3 Suppl 1): S7-S11

Bergeron C, Jeannel D, Poveda J, Cassonnet P, Orth G (2000) Human papillomavirus testing in women with mild cytologic atypia. Obstet Gynecol 95(6 Pt 1): $821-827$

Cuzick J, Szarewski A, Cubie H, Hulman G, Kitchener H, Luesley D, McGoogan E, Menon U, Terry G, Edwards R, Brooks C, Desai M, Gie C, Ho L, Jacobs I, Pickles C, Sasieni P (2003) Management of women who test positive for high-risk types of human papillomavirus: the HART study. Lancet 362: $1871-1876$

Guyot A, Karim S, Kyi MS, Fox J (2003) Evaluation of adjunctive HPV testing by hybrid capture II in women with minor cytological indicates that triage is acceptable to women, and the ability to return to recall not only $50 \%$ of those referred to colposcopy but also the $35 \%$ of women who were HPV-negative are both significant benefits (TOMBOLA Group, 2009a, b, c).

Although the HPV-positive rate in women with mild dyskaryosis is high, the negative predictive value of an HPV test in these women is over $96 \%$ (Moss et al, 2006), and the use of triage allows $16 \%$ of these women to be returned to routine recall.

The difference in HPV-positive rates between the centres involved in this project highlights the inter-laboratory variation in grade classification, which will be reflected in any wider rollout of HPV triage around the country, in terms of rates of referral to colposcopy. It also highlights the need for efficient management of HPV-positive women found to be negative at colposcopy. The PPV for CIN2 or worse, $16 \%$, was relatively low, which suggests that although HPV triage is a useful tool for returning women at low risk to routine recall, further refinement of triage should be considered to improve PPV without loss of sensitivity. This could exploit HPV genotyping and biomarkers, both of which have been shown to be capable of improving specificity (Szarewski et al, 2008), with the need to avoid increasing reporting times.

\section{ACKNOWLEDGEMENTS}

Professor HC Kitchener was supported by the Manchester Biomedical Research Centre. All authors have completed the Unified Competing Interest form at http://www.icmje.org/coi_disclosure. pdf (available on request from the corresponding author), and declare no financial relationships with any organisations that might have an interest in the submitted work in the previous three years and, no other relationships or activities that could appear to have influenced the submitted work. The Cancer Screening Evaluation Unit received funding from the NHS Cancer Screening Programmes to carry out this evaluation. The corresponding author has the right to grant on behalf of all authors and does grant on behalf of all authors, an exclusive licence (or nonexclusive for government employees) on a worldwide basis to the BMJ Publishing Group Ltd. and its licensees to permit this article (if accepted) to be published in BMJ editions and any other BMJPGL products and sub-licences to exploit all subsidiary rights, as set out in our licence (http://resources.bmj.com/bmj/authors/ checklists-forms/licence-for-publication). abnormalities for the diagnosis of $\mathrm{CIN} 2 / 3$ and cost comparison with colposcopy. BMC Infect Dis 3: 23

Howell-Jones R, Bailey A, Beddows S, Sargent A, de SN, Wilson G, Anton J, Nichols T, Soldan K, Kitchener H (2010) Multi-site study of HPV type-specific prevalence in women with cervical cancer, intraepithelial neoplasia and normal cytology, in England. Br J Cancer 103(2): $209-216$

Kitchener HC, Almonte M, Wheeler P, Desai M, Gilham C, Bailey A, Sargent A, Peto J (2006) HPV testing in routine cervical screening: cross sectional data from the ARTISTIC trial. Br J Cancer 95(1): 56-61

Legood R, Gray A, Wolstenholme J, Moss S (2006) Lifetime effects, costs, and cost effectiveness of testing for human papillomavirus to manage low grade cytological abnormalities: results of the NHS pilot studies. BMJ 332(7533): 79-85

Moss S, Gray A, Legood R, Vessey M, Patnick J, Kitchener H (2006) Effect of testing for human papillomavirus as a triage during screening for cervical cancer: observational before and after study. BMJ 332(7533): $83-85$

Munoz N, Castellsague X, de Gonzalez AB, Gissmann L (2006) Chapter 1: $\mathrm{HPV}$ in the etiology of human cancer. Vaccine 24(Suppl 3): S3/1-10

NHS (2009) Office for national statistics; cancer statistics registrations. Series MBI No.37, http://www.statistics.gov.uk/statbase/Product.asp?vlnk $=8843$ 
Peto J, Gilham C, Fletcher O, Matthews FE (2004) The cervical cancer epidemic that screening has prevented in the UK. Lancet 364(9430): $249-256$

Rebello G, Hallam N, Smart G, Farquharson D, McCafferty J (2001) Human papillomavirus testing and the management of women with mildly abnormal cervical smears: an observational study. BMJ 322(7291): $893-894$

Sargent A, Bailey A, Turner A, Alimonte M, Gilham C, Baysson H, Peto J, Roberts C, Thomson C, Desai M, Mather J, Kitchener H (2010) Optimal threshold for a positive hybrid capture 2 test for detection of human papillomavirus; data from the ARTISTIC trial. J Clin Microbiol 48(2): $554-558$

Sasieni PD, Cuzick J, Lynch-Farmery E (1996) Estimating the efficacy of screening by auditing smear histories of women with and without cervical cancer. The National Co-ordinating Network for Cervical Screening Working Group. Br J Cancer 73(8): $1001-1005$

Szarewski A, Ambroisine L, Cadman L, Austin J, Ho L, Terry G, Liddle S, Dina R, McCarthy J, Buckley H, Bergeron C, Soutter P, Lyons D, Cuzick J (2008) Comparison of predictors for high-grade cervical intraepithelial neoplasia in woman with abnormal smears. Cancer Epidemiol Biomarkers Prev 17(11): 3033-3042

\section{APPENDIX}

List of group members.

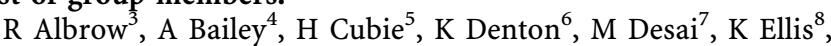
C Evans ${ }^{9}$, M Ferry ${ }^{10}$, A Fisher ${ }^{11}$, V Frew ${ }^{12}$, T Giles ${ }^{9}, \mathrm{R}$ HowellJones $^{13}, \mathrm{~K} \mathrm{Hunt}^{6}$, R Legood ${ }^{14}$, T Levine ${ }^{10}$, R Lonsdale ${ }^{12}$, J Marshall ${ }^{7}$, J Medlock $^{15}$, C Moore 5 , P Muir ${ }^{16}$, J Rimmer ${ }^{2}$, A Sargent ${ }^{4}$, JHF Smith ${ }^{8}$, $\mathrm{K}_{\text {Soldan }}{ }^{13}, \mathrm{~J} \mathrm{Tidy}^{8}$, L Turnbull ${ }^{9}$, A Turner ${ }^{17}$, P Walker ${ }^{18}$, R Winder ${ }^{2}$, M Young ${ }^{18}$

${ }^{3}$ School of Cancer Studies and Enabling Sciences, University of Manchester, Manchester Academic Health Science Centre, Manchester, UK; ${ }^{4} \mathrm{HPA}$ Manchester, Manchester, UK; ${ }^{5}$ Scottish
TOMBOLA Group (2009a) Biopsy and selective recall compared with immediate large loop excision in management of women with low grade cervical cytology referred for colposcopy: mulitcentre randomised controlled trial. BMJ 339: b2549

TOMBOLA Group (2009b) Cytological surveillance compared with immediate referral for colposcopy in management of women with low grade cervical abnormalities: multicentre randomised controlled trial. BMJ 339: b2546

TOMBOLA Group (2009c) Options for managing low grade cervical abnormalities detected at screening: cost effectiveness study. BMJ 339: b2549

Waterhouse J, Muir C, Correa P, Powel J eds. (1976) Cancer incidence in five continents. In International Agency for Research on Cancer, World Health Organisation, Vol. III, IARC Scientific Publication No 15: Lyon

cC)(2) This work is licensed under the Creative Commons

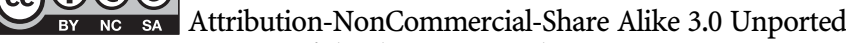
License. To view a copy of this license, visit http://creativecommons. org/licenses/by-nc-sa/3.0/

HPV Reference Laboratory, Edinburgh, UK; ${ }^{6}$ Southmead Hospital, Bristol, UK; ${ }^{7}$ Central Manchester University Hospitals NHS Foundation Trust, Manchester, UK; ${ }^{8}$ Sheffield Teaching Hospitals NHS Foundation Trust, Sheffield, UK; ${ }^{9}$ Royal Liverpool University Hospital, Liverpool, UK; ${ }^{10}$ North West London Hospitals NHS Trust, Harrow, UK; ${ }^{11}$ Queen Elizabeth Hospital, Gateshead, UK; ${ }^{12}$ Norfolk and Norwich University Hospital, Norwich, UK; ${ }^{13}$ Health Protection Agency, London, UK; ${ }^{14}$ London School of Hygiene and Tropical Medicine, London, UK; ${ }^{15}$ University Hospital Aintree, Liverpool, UK; ${ }^{16} \mathrm{HPA}$ South West Regional Laboratory, Bristol, UK; ${ }^{17}$ Manchester Royal Infirmary, Manchester, UK; ${ }^{18}$ Royal Free Hospital, London, UK. 